\title{
EL APRENDIZAJE Y SERVICIO SOLIDARIO EN EL ÁMBITO DE LA EDUCACIÓN INFANTIL. ESTADO DE LA CUESTIÓN
}

The service learning in the field of Early Childhood Education. State of the issue

O serviço de aprendizagem na área da Educação Infantil. Estado da questão

\section{Enrique Rivera García \\ Jonatan García-Suárez \\ $M^{a}$ Luisa López Raya}

Universidad de Granada, España. Fono: +34 958 249641. Correo electrónico: erivera@ugr.es

\section{Resumen}

Impulsar la responsabilidad social está siendo uno de los objetivos prioritarios de la universidad en estos últimos años. Dentro de este marco, podemos decir que el voluntariado ha alcanzado un nivel de consolidación aceptable, pero aún queda mucho camino por recorrer. Otra vía para la incorporación de la responsabilidad social en la universidad está siendo el Aprendizaje y Servicio Solidario, que avanza un paso más e incorpora como metodología de aprendizaje la vinculación de los aprendizajes académicos con el desarrollo de actividades con y para la comunidad. Este artículo se marca como objetivo ver la actual implantación del Aprendizaje y Servicio Solidario en la formación de docentes de Educación Infantil. Esta aproximación al estado de la cuestión se realiza desde una metodología descriptiva, pero ayudada con técnicas cualitativas facilitadas por el software de análisis de datos cualitativos Nvivo. Los principales resultados encontrados nos dicen que las competencias más desarrolladas en los estudiantes son las de carácter inter e intrapersonal, aunque también se muestran con menor intensidad las enfocadas al aprendizaje de contenidos específicos y su transformación en competencias sistémicas en el alumnado. La metodología de investigación que predomina en los documentos analizados es descriptiva, con poca presencia cualitativa. En conclusión, podemos decir que se está iniciando un camino, especialmente el de la investigación, y que aún no existe una masa crítica con autores relevantes ni publicaciones específicas en el campo.

Palabras clave: responsabilidad social, formación del profesorado, análisis cualitativo de datos, software Nvivo. 


\begin{abstract}
Fostering social responsibility is one of the main aims of university in later years. Within this context, it can be stated that voluntary service is being progressively consolidated, however, there is still a long way to go. Another way to incorporate social responsibility in the university is the Service Learning, which goes a step further by adding the development of community work as learning methodology. This paper aims to see the actual implementation of Service Learning and volunteering in the training of Pre-school Education teachers. This approach is made through a descriptive methodology, helped by qualitative techniques provided by the Qualitative Data Analysis Software. Nvivo. The main findings show that the most developed competences in the students have inter and intrapersonal character. With a lower intensity, those focused on learning specific content and its transformations in systemic competences are also shown in the students. The main methodology of the analyzed documents is descriptive, with little presence of qualitative methodology. As a conclusion, only recently research, has begun; there is no critical mass of relevant authors or specific publications in this field yet.
\end{abstract}

Keywords: social responsibility, teacher training, qualitative data analysis, software Nvivo.

\title{
Resumo
}

Promover a responsabilidade social ainda é uma das prioridades da universidade nos últimos anos. Neste contexto, podemos dizer que o voluntariado atingiu um nível aceitável de consolidação, mas ainda há muito a ser feito. Outra maneira de incorporar a responsabilidade social na universidade ainda é o aprendizado de serviço, que vai um passo além e incorpora metodologia de aprendizagem que liga o aprendizado acadêmico para o desenvolvimento de actividades com e para a comunidade. Este artigo tem objetivo de ver a implementação real da formação de professores de aprendizagem serviço na Educação Infantil. Esta abordagem para o estado de coisas é feita a partir de uma metodologia descritivo, mas ajudou com técnicas qualitativas fornecidos pelo software NVIVO análise de dados qualitativos. Os principais resultados dizem-nos que as habilidades mais desenvolvidas de estudantes são de caráter inter e intrapessoal, embora com menor intensidade está focada em aprender o conteúdo específico e sua transformação em 
habilidades sistêmicas nos estudantes. A metodologia de pesquisa que prevalece nos documentos analisados é descritiva, com pouca presença qualitativa. Em conclusão, podemos dizer que está começando no caminho, especialmente a investigação, e que há uma massa crítica com autores relevantes ou publicações específicas no domínio ainda.

Palavras-chave: responsabilidade social, formação de profesores, análise qualitativa dos dados, Nvivo.

\section{Introducción}

Entender el Aprendizaje Servicio (ApS), pasa primeramente por realizar una visión global de lo que ha significado en estos últimos lustros la relación creada entre Ciencia y Sociedad. En un primer estadio de la misma nos encontramos con una idea de relación unidireccional, en el que la Sociedad se sitúa en un papel de receptora de conocimiento frente a la Ciencia, que, a través de la investigación básica, va a ser quien se ocupe y preocupe de proveer de conocimiento a la primera. Este modelo de Ciencia conocido en la década de los setenta como "Science pusch", viene a generar un pensamiento social de visualizarla como la garantía y solución de los problemas globales que afectan a la Sociedad. Al ser un modelo unidireccional que sitúa a la Ciencia un escalón por encima, le otorgaba el poder de decisión sobre el "qué, cómo y para qué” investigar. Posteriormente se abre paso una segunda visión de la relación Ciencia y Sociedad, en la que esta última comienza a invertir la relación inicial para dejar paso a una visión más específica; se buscan aportaciones científicas que solucionen problemas tecnológicos, económicos y sociales; “Demand pull". A partir de este momento será la demanda de los mercados los que determine la ciencia (Herrero, 2010).

Frente a los modelos descritos anteriormente e identificados por Gibbons, Limoges, Mowotny, Schwartzman, Scott y Trow (1994) como "Modo 1", comienza a emerger otra forma de entender la relación entre ciencia y sociedad: "Modo 2". En él y siguiendo a Israel, Schultz, Parker y Becker (1998) se pretende pasar de las relaciones verticales expresadas en el anterior modelo, a una propuesta de horizontalidad, donde la premisa inicial es que el conocimiento se construye desde y con la comunidad, reconociendo sus necesidades y facilitando la participación de todos los implicados en el 
proceso. Además, se pretende que el conocimiento generado sea útil para solucionar problemas desde la construcción de un proceso de investigación cíclico y permanente, cercano a la propuesta de investigación en la acción (Carr y Kemmis, 1988; Ebbutt, y Elliott, 1990; Freire 1990; Gollete y Lessard-Hébert, 1988; Kemmis y McTaggart, 1988; Lewin, 1946). Por último, si investigamos para, con y desde la comunidad, la información producida debe ser contenida en un lenguaje que facilite su difusión entre todos.

Una vez aclarado el binomio Ciencia-Sociedad, y posicionados en el "Modo 2" como andamiaje para construir conocimiento útil a los intereses de la comunidad con la que deseamos trabajar, debemos entrar en tres conceptos claves que generan mucha controversia a la hora de plantear el Aprendizaje y Servicio Solidario (A+SS): 'Voluntariado', 'Aprendizaje Servicio' y 'Aprendizaje Servicio Solidario'. Su propio enunciado ya nos sugiere una gradación clara de estos tres conceptos con una gran relación entre sí. De hecho el problema no está tanto en su definición sino establecer las finas barreras que los separan.

El voluntariado es un concepto más conocido y universal, vinculado a la responsabilidad social que toda universidad debiera tener adquirida. Su finalidad se focaliza en ofrecer un servicio a la comunidad, especialmente en colaboración con aquellos colectivos agrupados en organizaciones de carácter no gubernamental. Evidentemente existe aprendizaje, pero más enfocado al desarrollo de capacidades de carácter intra e interpersonal, muy relacionadas con actividades prosociales que tienen por objeto el desarrollo de competencias sociales y ciudadanas (Campo, 2014).

En cuanto al Aprendizaje Servicio podríamos encontrar sus antecedentes a finales del siglo XIX y principios del XX, en los que podemos situar los primeros movimientos de la pedagogía activa, que fundamenta su propuesta desde el principio de aprender desde el 'hacer' (Dewey, 1916. Cit. en Campos, 2014). En la búsqueda de una educación integral, se pretende vincular los aprendizajes tanto al ámbito cognitivo, el procedimiental y el actitudinal, situando el énfasis especial en el segundo como motor primordial, pero estableciendo un trabajo en bucle en el que la experiencia vivida nos llevará al descubrimiento de la teoría que la sustenta desde un ejercicio reflexivo. Si buscamos en la 
literatura definiciones de Aprendizaje y Servicio podemos encontrar cientos, por esta razón vamos a quedarnos con las ideas claves que lo definen dentro del ámbito universitario. Es un método de enseñanza que tiene por finalidad poner en conexión el servicio a la comunidad con la formación académica, $\mathrm{Su}$ objetivo principal se centra en provocar el aprendizaje desde la experiencia vivida en contextos de realidad a todos los niveles: cognoscitivo, intrapersonal e interpersonal (Campos, 2014; Jacoby, 1996 y Puig y Palos, 2006). Para aclarar mejor las diferencias entre lo que podemos entender como 'Voluntariado' y 'Aprendizaje y Servicio', veamos en la figura 1 de forma ilustrativa y en forma de cuadrante las diferencias entre ambos conceptos:

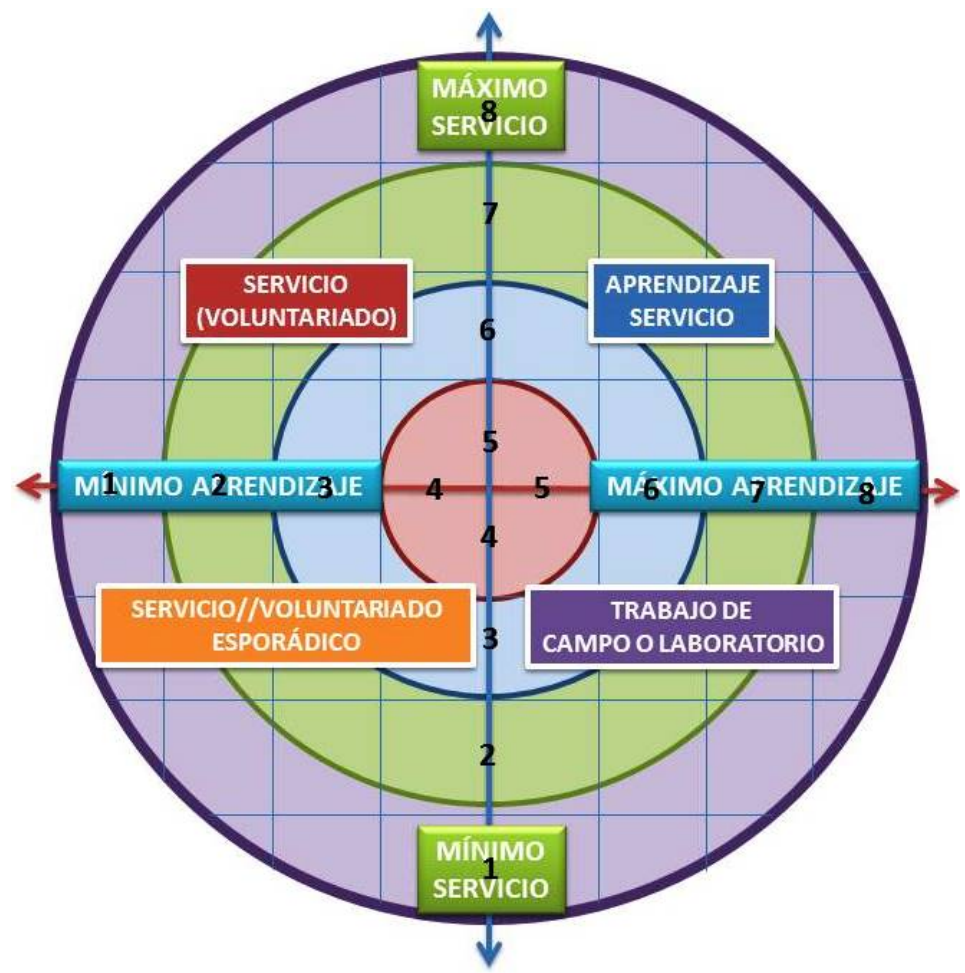

Figura 1. Cuadrante del aprendizaje servicio. Adaptado de Service Learning 2000 Center (1996)

En la Figura 1 podemos ver de forma sintética las grandes diferencias entre cada uno de los conceptos. Cuando hablamos de Voluntariado, podemos ver cómo el servicio a la comunidad es alto situándose en la franja (4-8), pero a cambio, el aprendizaje deja de tener importancia y pasa a ser un efecto colateral y no controlado de forma intencionada 
por el docente. Generalmente se provocan aprendizajes de carácter prosocial, aunque cabe la posibilidad del desarrollo de competencias de carácter sistémico y específico.

En el caso del Aprendizaje Servicio, cambia el enfoque y ambos ejes (Servicio y Aprendizaje) se sitúan en valores de la franja alta (4-8), ya que la intencionalidad es doble: por una parte se pretende lograr los objetivos -a los que podemos llegar desde el voluntariado-, pero siempre dentro del contexto de una asignatura o materia que, además de la adquisición de competencias prosociales, pretende claramente vincular la experiencia con la adquisición de aprendizajes vinculados a los aspectos conceptuales y procedimentales de la asignatura y su manifestación en competencias por parte del estudiante de carácter sistémico.

En cuanto a los cuadrantes inferiores de la Figura 1, se pretende dejar claro que todo lo que signifique la realización de trabajos de campo, como pueden ser las prácticas de enseñanza en un pregrado, quedarían fuera de lo que entendemos como Aprendizaje Servicio, aunque sí serían susceptibles de ser enfocadas dentro de esta metodología. Por último, el último cuadrante hace referencia a actuaciones puntuales de voluntariado (organización de un evento lúdico en un centro educativo, un taller específico, etc.), que al no mantenerse en el tiempo pierden gran parte de su valor y aportan poco a la comunidad y a los estudiantes.

El concepto 'Aprendizaje y Servicio' vendría a ser una traducción literal del término anglosajón 'Service Learning', pero en el contexto latinoamericano podemos identificar el mismo concepto como Aprendizaje y Servicio Solidario (A+SS). Particularmente nos parece más completo este término y nos sentimos más identificados con el mismo, ya que el lado de la 'solidaridad' cobra especial relevancia. Por esta razón nos alejamos del concepto anglosajón, coincidiendo con Tapia (2010: 26) en una pedagogía del "hacer juntos" frente al "dar para" más ligado a la cultura anglosajona del voluntariado. A partir de ahora hablaremos de Aprendizaje-Servicio Solidario (A+SS), entendido como una pedagogía y metodología de trabajo en el ámbito educativo (Kendall y Associates, 1990; Puig, 2007 y Staton, 1990) que va a estar sustentada por tres ideas claves (Tapia, 2010: 28): 
- Aprendizaje activo. Donde el estudiante será el protagonista del proceso, junto a la comunidad receptora de la intervención. Ellos serán quienes detecten las necesidades, diseñen el proyecto, ejecuten la propuesta de intervención, realicen la evaluación y reinicien el ciclo desde el conocimiento generado y las nuevas demandas que surjan.

- Compromiso social. Las propuestas de intervención serán preferentemente pensadas y construidas para contextos de riesgo de exclusión social. Las propuestas irán dirigidas a solucionar problemas reales y surgirán de las demandas de los propios receptores de la intervención.

- Comunidad de aprendizaje. El aprendizaje será mutuo. Los estudiantes universitarios se beneficiaran de poder trabajar en contextos reales, aprendiendo desde la búsqueda de soluciones a problemas cotidianos que se encontrarán en su futuro desarrollo profesional. Los receptores del servicio solidario (miembros de la comunidad objeto de la intervención), tendrán la oportunidad de crecer como personas y futuros ciudadanos.

El objeto de esta investigación se centra en posicionar la presencia del Aprendizaje y Servicio Solidario en el ámbito de la formación de los docentes de Educación Infantil. Entendemos que es un ámbito ideal para incorporar esta metodología de trabajo, especialmente en aquellos contextos que presentan mayor desigualdad social. En base a este objeto de investigación nos hemos planteado alcanzar los siguientes objetivos:

- Revisar y recopilar en las bases de datos más significativas del ámbito de la investigación de Ciencias Sociales, los artículos relacionados con investigaciones o experiencias de A+SS en el ámbito de la formación de docentes de Educación Infantil.

- Organizar los documentos encontrados en base a criterios de contenidos presentes.

- Analizar críticamente los documentos localizados para establecer un estado de la cuestión inicial sobre el tema.

\section{Metodología}

El estudio realizado es fundamentalmente de carácter descriptivo, aunque también hemos entrado a un análisis cualitativo a partir de los resúmenes de los artículos seleccionados. Para la localización de los documentos con los que hemos trabajado, hemos optado por 
realizar una búsqueda de información en tres bases de datos habituales para la investigación en el campo de las Ciencias Sociales: Scopus, Web of Science y Dialnet. Las dos primeras para la localización de artículos en inglés y la última para aquellos escritos en castellano. Las palabras claves utilizadas en las búsquedas han sido: 'Aprendizaje', 'Servicio', 'Universidad', 'Educación Infantil' y 'Service Learning' y 'Preschool' en inglés. Como complemento a estas búsquedas hemos utilizado también una base de datos de la red de Aprendizaje Servicio Universitario en Educación Física de reciente creación. Las búsquedas realizadas las hemos centrado en los diez últimos años (2005-2015), con la finalidad de acotar y seleccionar los documentos en base a criterios de actualidad.

\begin{tabular}{|c|c|c|c|c|c|c|c|}
\hline Autor 1 & Autor 2 & Autor 3 & año & Fuente & Localización & Título & Resumen \\
\hline Amat, A. F. & Moliner, L. & & 2010 & REIFOP & $13,(4)$ & El Aprendiza & endizaje Ser \\
\hline Aramburuzabal & García, P. & & 2012 & V II CIDUI & & EL APRENDIZ & En esta coms \\
\hline Bartolomé, S. J. & & & 2013 & Journal of $\mathrm{M}$ & Volume 23,1 & Growing Thr & The purpose \\
\hline Capella, C. & Gil, J. & Martí, M. & 2014 & Apunts & n.. $116,2.0 \mathrm{t}$, & La metodolo & presenta los \\
\hline Capella, C. & Gil, J. & Martí, M. & 2015 & Profesorado & Vol. 19, № 1 & Estudio de ce & Este trabajo \\
\hline Celma, M. & & & 2014 & Universitat $\mathrm{B}$ & http://www & Proyecto Art & El 'Encuentrd \\
\hline Chiva, $\mathrm{O}$ & Gil, J. & Hernando, C & 2014 & Tándem & 44 p. $41-48$ & Innovación n & Este artículo \\
\hline Cruz, E. & & & 2014 & Universitat $\mathrm{R}$ & http://www & Trabajo Fin & El estudiant \\
\hline Davis, E. & & & 2006 & Teaching and & $22281-301$ & Characterizir & Effective tea \\
\hline Eyler, J.S. & Giles, J. D. & Stenson C. M & 2001 & nderbilt Uni & versity $A$ & At A Glance: & What We Knc \\
\hline
\end{tabular}

Figura 2. Ejemplo de la base de datos construida a partir de las fuentes localizadas

Una vez localizados todos los documentos con una relación directa o indirecta con la Educación Infantil y la formación de docentes y profesionales relacionados con estas edades (0-6 años), se ha procedido a construir una base de datos en formato Excel (Figura 2), que una vez importada al software NVivo de análisis cualitativo, nos ha permitido obtener los resultados que se muestran.

Para el análisis cualitativo hemos partido en primer lugar de los títulos y los resúmenes de los documentos indexados (se han traducido al castellano los escritos en inglés). Para ello hemos realizado el análisis en dos niveles. En el primero, se ha trabajado con los conceptos presentes en título y resumen, utilizando tres estrategias básicas con el NVivo: consulta de frecuencia de palabras (en ambos apartados con agrupación de conceptos que comparten la misma raíz), realización de una marca de nube para que 
visualmente se puedan ver los conceptos más significativos y cerramos este análisis con un árbol de palabras de cada uno de los términos seleccionados para buscar las relaciones entre ellos. En el segundo nivel se ha realizado una indexación mixta en base a conceptos emergentes y previos de los documentos analizados, para posteriormente, desde la construcción de una matriz de intersección, ver las categorías con mayor presencia a nivel global y particular de cada fuente utilizada.

\section{Análisis y discusión de resultados}

En una primera aproximación a la base de datos creada, podemos ver en la Gráfica 1 que existe una gran dispersión de autores. De los 65 presentes en los estudios y trabajos encontrados, el 91\% figura en una sola publicación, un 6\% figuran en dos publicaciones, un $2 \%$ en tres y un $1 \%$ en cuatro. Esto nos ofrece una idea del alto nivel de dispersión y la escasa especialización que existe en este campo específico del A+SS.

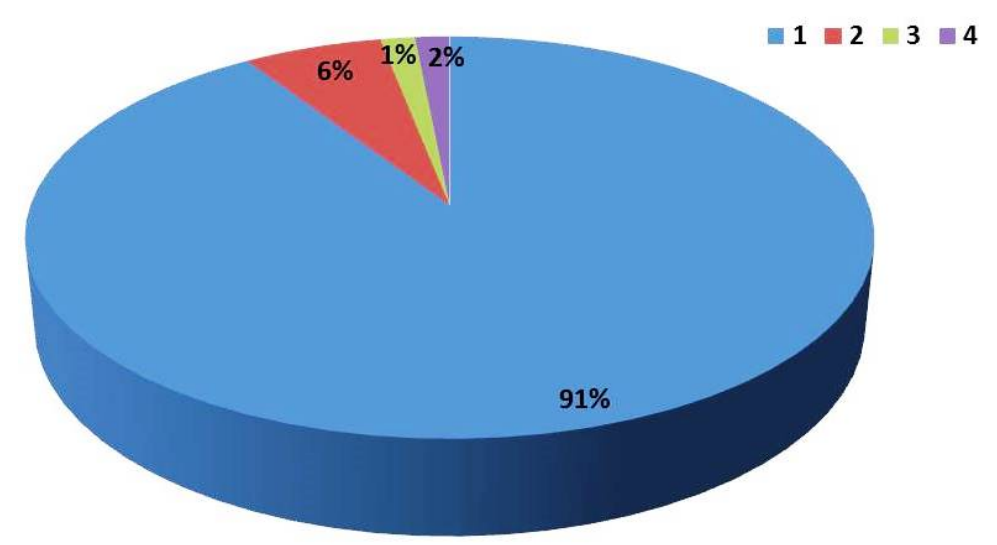

Gráfica 1. Porcentaje de referencias encontradas por autores

Si nos detenemos en un análisis en base a los años de publicación, podemos observar en la Gráfica 2, cómo el mayor número de publicaciones se concentra en estos últimos cinco años, momento en el que cobra auge la incorporación de esta metodología al ámbito universitario. La evolución es constante y se aprecia un claro interés por la aplicación de esta metodología en el ámbito de la formación del docente de Educación 
Infantil. A pesar de todo debemos ser conscientes del bajo nivel de publicaciones, especialmente en revistas científicas sobre el tema.

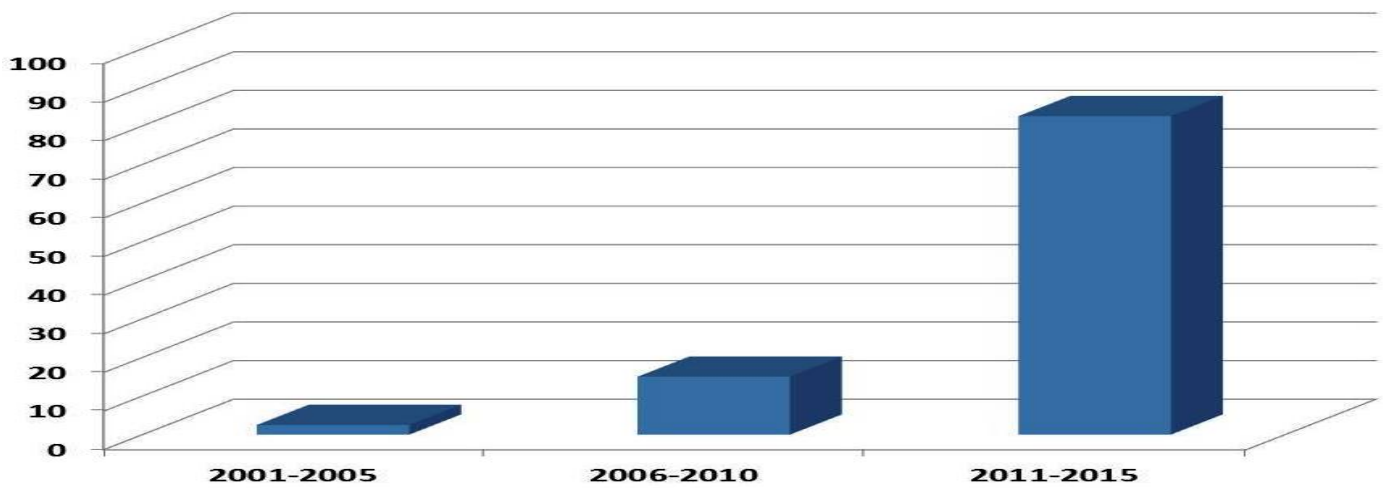

Gráfica 2. Porcentaje de producción en los últimos 15 años sobre la temática

Si nos detenemos en la Tabla 1, podemos ver que el total de publicaciones que acogen temáticas relacionadas con el $\mathrm{A}+\mathrm{SS}$ en relación con la Educación Infantil son veintitrés. Llama la atención el número de referencias dentro del ámbito de la Salud que han aparecido y debemos tener presente, que aunque directamente no se centran en formación de docentes, si realizan sus experiencias en la etapa de 0-6 años. Queremos hacer notar que solo una única publicación acoge 13 referencias educativas, y pertenecen a experiencias publicadas por la Universita Rovira y Virgili, que formalmente no es una revista de investigación, pero que hemos creído conveniente tener en cuenta por ser referencias de alta significatividad para la formación de docentes de Educación Infantil.

Tabla 1. Distribución de fuentes en base a Área de Conocimiento y $\mathbf{n}^{\circ}$ de referencias en Publicación

\begin{tabular}{|l|c|c|c|c|c|}
\hline \multicolumn{2}{|c|}{} & \multicolumn{4}{c|}{ Revistas (23) } \\
\hline Área de Conocimiento & No artículos & 1 referencia & 2 referencias & 3 referencias & 13 referencias \\
\hline Salud & $\mathbf{5}$ & 5 & & & \\
\hline CC. Sociales & $\mathbf{8}$ & 5 & & 1 & \\
\hline Educación & $\mathbf{2 7}$ & 9 & 2 & & 1 \\
\hline
\end{tabular}

Una vez realizada esta primera aproximación a las fuentes encontradas en relación a la temática, vamos a pasar a un análisis de carácter más cualitativo tomando como base dos aspectos: el título del documento y el resumen del mismo. Desde ellos vamos a tratar de analizar las principales temáticas que aparecen y el enfoque metodológico en el que se han apoyado. 


\subsection{Análisis de los conceptos más significativos aparecidos en título y resumen}

Como se explicaba anteriormente en el epígrafe de la metodología, se han localizado los conceptos más relevantes (Tabla 2) y de ellos hemos pasado al análisis de aquellos que mayor grado de significatividad pueden tener de cara a establecer relaciones entre ellos.

Tabla 2. Conceptos más significativos localizados en los títulos de los documentos.

\begin{tabular}{|c|c|c|c|c|c|c|c|c|}
\hline Palabra & Longitud & Conteo & Palabra & Longitud & Conteo & Palabra & Longitud & Conteo \\
\hline educación & 9 & 12 & evaluación & 10 & 3 & examen & 6 & 2 \\
\hline enseñanza & 9 & 5 & grado & 5 & 3 & familia & 7 & 2 \\
\hline maestros & 8 & 5 & infancia & 8 & 3 & fin & 3 & 2 \\
\hline escuela & 7 & 5 & infantil & 8 & 3 & guiada & 6 & 2 \\
\hline comunidad & 9 & 4 & primaria & 8 & 3 & medicina & 8 & 2 \\
\hline estudiantes & 11 & 4 & primera & 7 & 3 & metodología & 11 & 2 \\
\hline experiencia & 11 & 4 & reflexión & 9 & 3 & mujeres & 7 & 2 \\
\hline formación & 9 & 4 & universidad & 11 & 3 & practicum & 9 & 2 \\
\hline física & 6 & 4 & conocimiento & 12 & 2 & pre & 3 & 2 \\
\hline lectoescritura & 14 & 4 & cooperación & 11 & 2 & productiva & 10 & 2 \\
\hline lenguas & 7 & 4 & desarrollo & 10 & 2 & profesorado & 11 & 2 \\
\hline niños & 5 & 4 & didáctica & 9 & 2 & programa & 8 & 2 \\
\hline través & 6 & 4 & docentes & 8 & 2 & proyecto & 8 & 2 \\
\hline ciudadanía & 10 & $\overline{3}$ & estrategia & 10 & 2 & trabajo & 7 & 2 \\
\hline práctica & 8 & 3 & estudio & 7 & 2 & 19932000 & 8 & 1 \\
\hline
\end{tabular}

Revisando las palabras aparecidas, hemos seleccionado 10 conceptos claves que entendemos nos pueden ayudar a descifrar las principales temáticas abordadas en los documentos analizados. En la marca de nube que se presenta podemos ver de forma gráfica los valores plasmados en la tabla 2. De ellos, hemos optado por seleccionar los siguientes conceptos para un posterior análisis de mayor profundidad: Educación; Enseñanza; Comunidad; Formación; Ciudadanía; Evaluación; Reflexión; Cooperación; Didáctica y Metodología. 


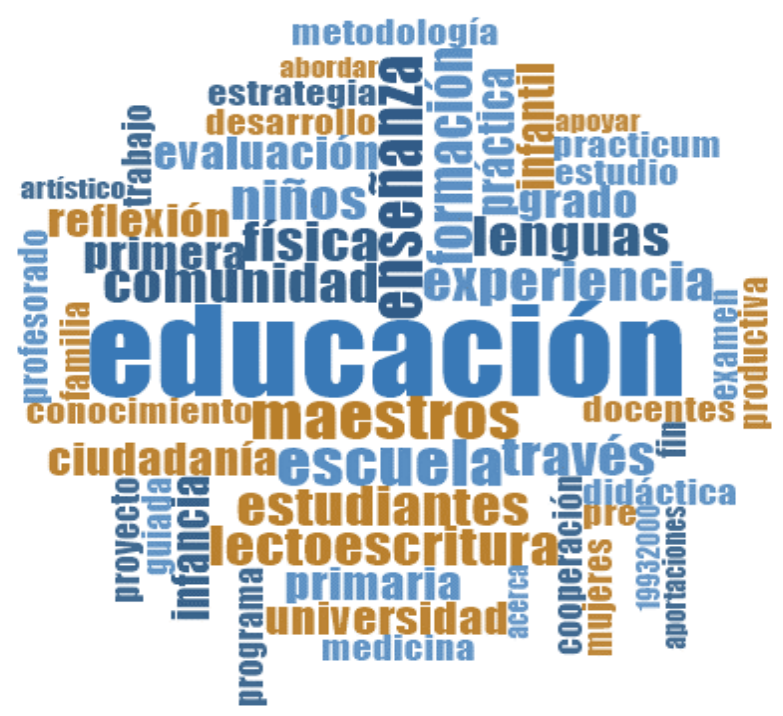

Figura 3. Marca de Nube de conceptos claves en título

Pasemos, aprovechando la oportunidad que nos ofrece el NVivo, a revisar las relaciones que se establecen en cada uno de los conceptos anteriores:

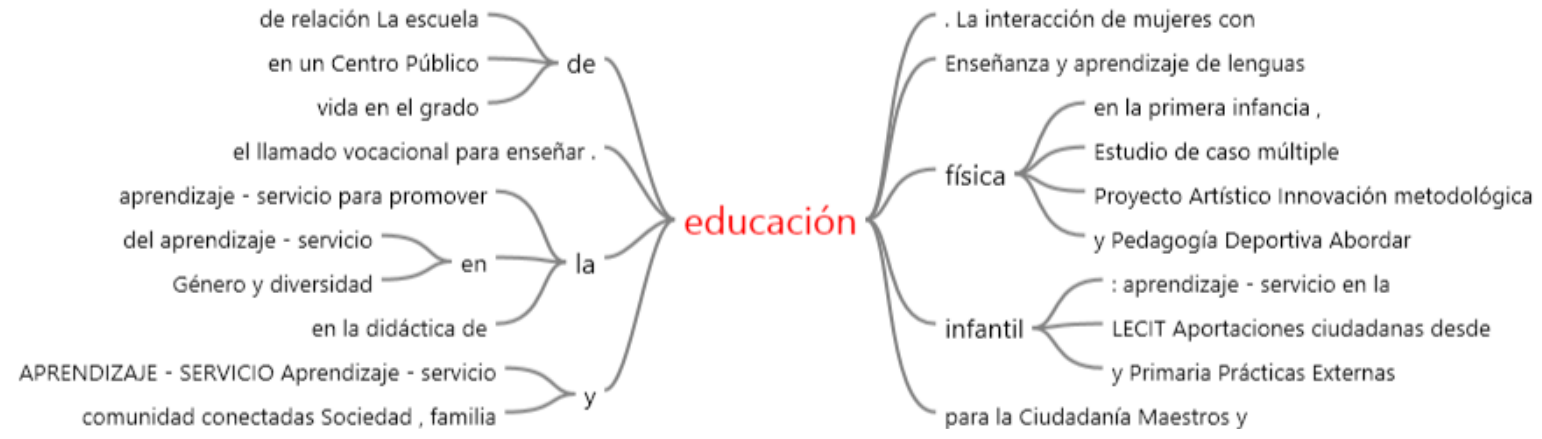

Figura 4. Árbol de palabras de "Educación" en el título

El árbol que presenta mayor ramificación es el que se relaciona con el concepto 'Educación', desde él podemos observar en la Figura 4, como se vincula claramente con ideas básicas como educación pública, la vocación del enseñante, el A+SS con una finalidad clara: favorecer la educación desde una perspectiva global, en la que no sólo se valore la visión integral, sino que se entienda como un objetivo de toda comunidad, en la que todos deben estar implicados: apoderados, docentes, estudiantes. 


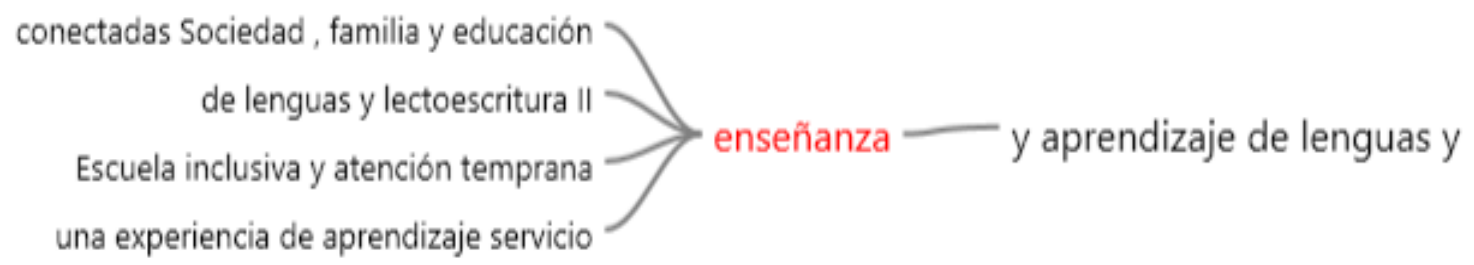

Figura 5. Árbol de palabras de "Enseñanza" en el título

Por otra parte, entrando en el análisis del concepto 'Enseñanza' (Figura 5), emergen otra serie de vínculos hacia enfoques educativos en los que los aprendizajes específicos cobran un papel significativo, en este caso centrado en el ámbito de la enseñanza de la lengua y la lectoescritura.

En cuanto al concepto 'Comunidad' se vincula, como no podría ser de otra manera, con la idea de un $\mathrm{A}+\mathrm{SS}$ en relación con la sociedad, la familia y el ámbito educativo. Es significativo cómo el concepto 'Formación', se centra en el alumnado universitario, proponiendo una mirada de ciudadanía crítica. Esta misma orientación se repite en el concepto 'Ciudadanía'. La idea de evaluación no se llega a concretar, por lo que el árbol de palabras que ofrece carece de sentido. No ocurre igual con el concepto 'Reflexión', que vinculado con el A+SS se entiende como una herramienta básica para su puesta en práctica.

En cuanto a los conceptos: 'Cooperación', 'Didáctica' y 'Metodología' el árbol de palabras que nos ofrece cada uno de ellos no presenta ninguna peculiaridad digna de resaltar.

Para cerrar esta aproximación a las fuentes analizadas, hemos realizado una 'Marca de Nube' (Figura 6) con la información de los documentos existentes en los resúmenes de los mismos. 


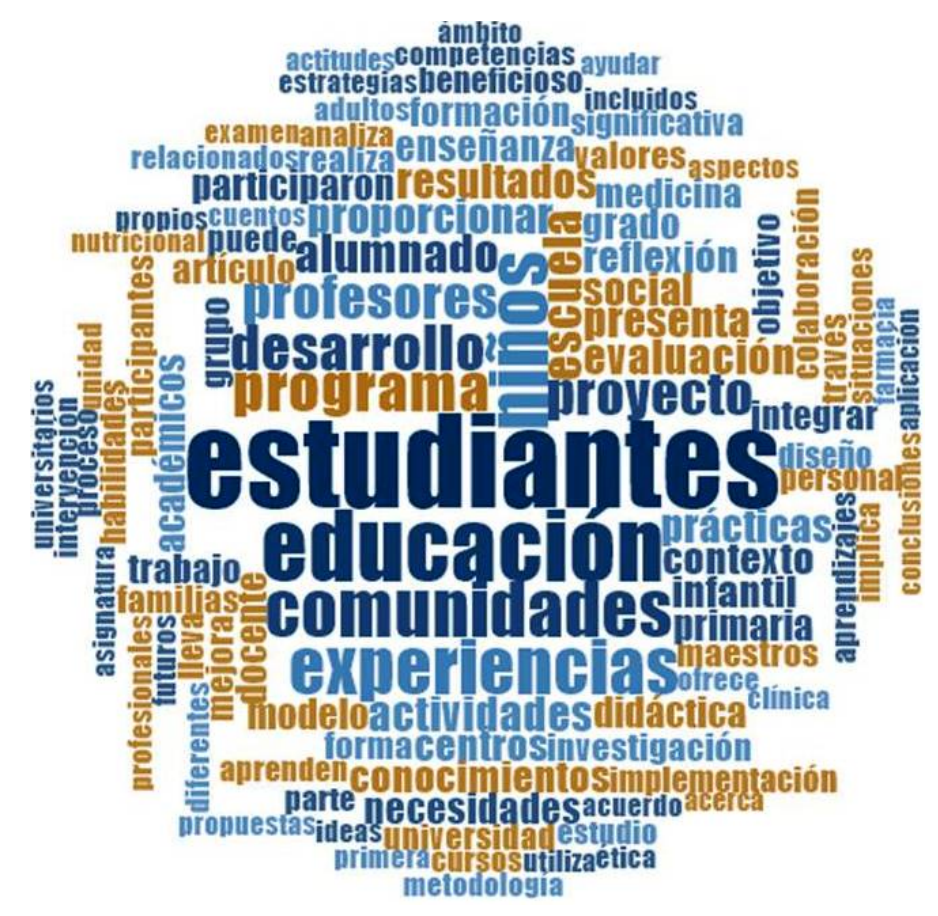

Figura 6. Marca de nube de conceptos principales del resumen de la fuente

En ella podemos observar los conceptos que los autores utilizan de forma más reiterada y que construyen el corpus del conocimiento generado sobre el A+SS, en el ámbito de la formación de los docentes de Educación Infantil. El de mayor relevancia es el de 'Estudiantes'; podemos inferir de este punto que las experiencias desarrolladas llevan por objetivo principal el dotar de herramientas y capacidades al alumnado universitario participante a través de la vivencia en contextos de realidad de sus experiencias formativas.

De alguna forma los tres conceptos que con más fuerza se manifiestan: educación, comunidades y experiencia así lo corroboran. Pero no perdamos de vista, aunque se muestra en vertical en la figura, el concepto 'Niños', lo que interpretamos como la lógica de entender que sin ellos, el A+SS no tendría sentido. La visualización de que comienza a atisbarse el paso de la experiencia a la investigación, lo podemos encontrar en la presencia de conceptos como: resultados, evaluación, investigación, metodología (aunque este concepto podría tener una doble interpretación) o conclusiones. A pesar de ello, aún se evidencia una escasa intensidad de presencia y debemos hacer mención que conceptos claramente relacionados con el diseño o la metodología de la investigación queda ausente 
de esta nube de palabras. Esto nos da pie para reafirmarnos en el bajo perfil que presenta la investigación en este campo de trabajo.

\subsection{Análisis de los documentos en base a objetivos, contenidos y metodología que plantean}

El estudio que abordamos se realiza para visualizar los enfoques principales que predominan en los documentos revisados. Como podemos observar en la Gráfica 3, si nos detenemos en los objetivos que se tratan de lograr, hay un claro predominio de aquellos que se enfocan hacia el logro de mejoras en competencias de carácter transversal $(38,5 \%)$, aunque no es nada desdeñable la evidencia de una clara intencionalidad hacia la consecución de capacidades muy relacionadas con los contenidos específicos de la materia (27\%). Lejos de estos valores se muestran objetivos orientados hacia las competencias de carácter sistémico, con una clara aplicabilidad del conocimiento (7,7\%). También debemos tener presente que hay documentos cuya única intencionalidad es la descripción de la experiencia realizada (11,5\%). Llama la atención de aquellas propuestas que se decantan al desarrollo del $\mathrm{A}+\mathrm{SS}$ en contextos de vulnerabilidad social $(11,5 \%)$ o con niños que presentan algún tipo de discapacidad motora, sensorial o psíquica $(3,8 \%)$. 


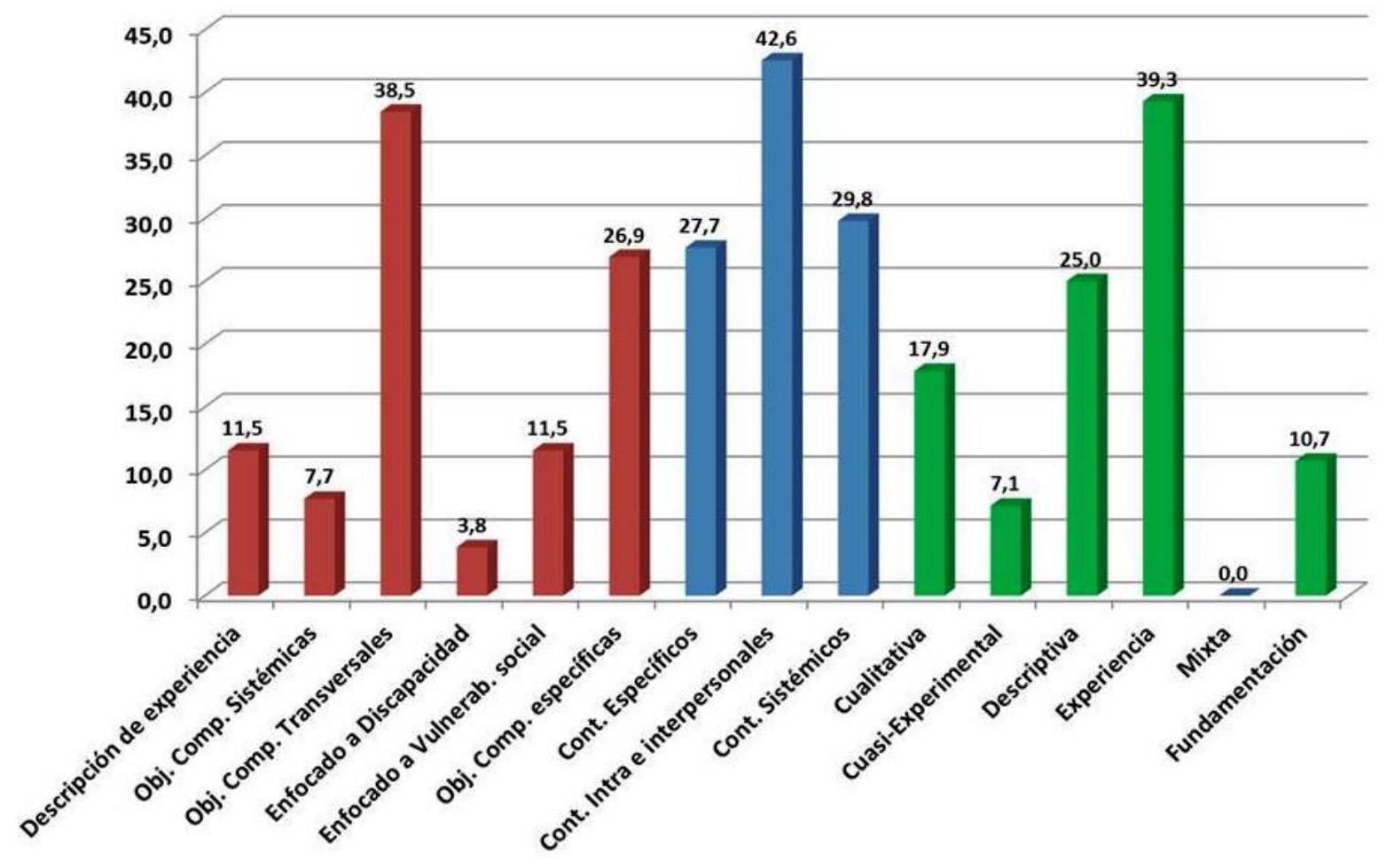

Gráfica 3. Análisis porcentual de los principales elementos de los documentos analizados

Si el análisis lo focalizamos en los contenidos que se trabajan en las experiencias que se describen, al igual que ocurría con los objetivos, son los relacionados con el desarrollo de competencias intra e interpersonales los que destacan con un $42,6 \%$ del total. En este caso, al contrario de lo que ocurría al marcar objetivos, los de carácter específico y sistémico prácticamente se sitúan en valores muy similares, $27,7 \%$ y $29,8 \%$ respectivamente.

Para finalizar esta primera aproximación a las fuentes localizadas, vamos a entrar en las tipologías y metodología de investigación presente. Es significativo que casi el 40\% de ellas se limitan a plantear las experiencias realizadas, incorporando en todo caso una breve evaluación realizada. En segundo lugar, predominan metodologías con diseños descriptivos basados en la aplicación de cuestionarios (25\%), aspecto que llama la atención cuando tanto los objetivos como los contenidos pretendidos se enfocan hacia aspectos fundamentalmente de carácter prosocial. Los enfoques de metodología cualitativa (17,9\%) nos parecen insuficientes basándonos en la argumentación anterior, máxime si tenemos en 
cuenta que son estudios y experiencias con un alto contenido centrado en las percepciones de los participantes. Por último mencionar la presencia de artículos $(10,7 \%)$ centrados en fundamentar el A+SS y establecer las bases para su trabajo.

Entrando en un análisis de mayor detalle y partiendo de la matriz realizada con el software NVivo (Tabla, 3), vamos a profundizar en cada una de las fuentes y enfoques realizados. En el planteamiento de los objetivos, vimos anteriormente que la fuerza se situaba en la búsqueda del logro de objetivos de carácter transversal, muy centrados en la adquisición de competencias intra e interpersonales; es el caso de los trabajos de Folco (2013), García (2011), Knutson y González (2010), Lake y Jones (2008), Maynes, Hatt, y Wideman, (2013), Páez y Puig, J. (2013), Vicens (2014) y Winterbottom, Vickie y Lake (2013). En todos ellos aparece la mejora personal, el servicio a la comunidad, la ética, la construcción de una conciencia social, en algún caso hasta planteado desde enfoques críticos. Sí queremos llamar la atención en una capacidad básica en el ámbito del A+SS: la reflexión. Páez y Puig (2014: 17) consideran que es clave para poder multiplicar el aprendizaje y poder dotarle del enfoque inter e intrapersonal que deben tener este tipo de experiencias. Pero debemos tener en cuenta que si no canalizamos adecuadamente este proceso, lo sistematizamos y le otorgamos el rigor necesario, nos podemos encontrar con una reflexión laxa, más cercana al recuerdo o resumen de la experiencia vivida que al análisis del proceso desarrollado. 
Tabla 3. Matriz de intersección de análisis de fuentes

Autores

\begin{tabular}{|c|c|c|c|c|c|c|c|c|c|c|c|c|c|c|c|}
\hline Autores & 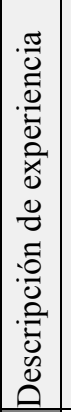 & 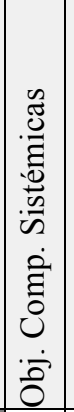 & 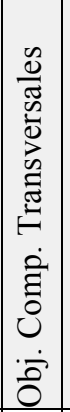 & 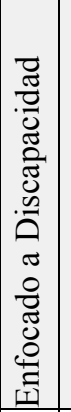 & 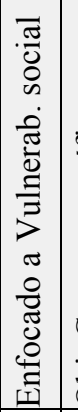 & 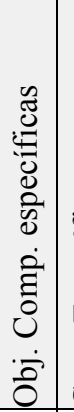 & $\begin{array}{c} \\
\\
0 \\
0 \\
0 \\
0 \\
0 \\
0 \\
0 \\
1 \\
\dot{0} \\
\dot{0} \\
0\end{array}$ & 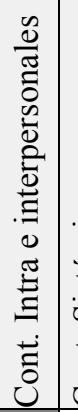 & 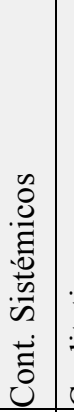 & 苞 & 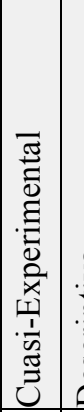 & 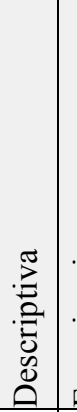 & 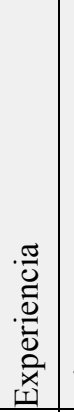 & $\frac{\pi}{x}$ & 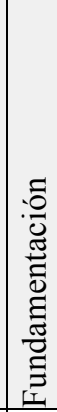 \\
\hline Amat, A. F.; Moliner, L. (2010) & 1 & 0 & 0 & 0 & 0 & 0 & 0 & 1 & 0 & 0 & 0 & 0 & 0 & 0 & 0 \\
\hline Aramburuzabala, P.; García, P. (2012) & 1 & 0 & 0 & 0 & 0 & 0 & 0 & 1 & 0 & 0 & 0 & 0 & 0 & 0 & 0 \\
\hline Bartolomé, S. J. (2013) & 1 & 0 & 0 & 0 & 0 & 0 & 0 & 1 & 0 & 1 & 0 & 0 & 0 & 0 & 0 \\
\hline Capella, C.; Gil, J.; martí, M. (2014) & 0 & 0 & 0 & 0 & 0 & 0 & 1 & 1 & 1 & 1 & 0 & 0 & 0 & 0 & 0 \\
\hline Capella, C.; Gil, J.; martí, M. (2015) & 0 & 0 & 0 & 0 & 0 & 0 & 0 & 1 & 0 & 1 & 0 & 0 & 0 & 0 & 0 \\
\hline Celma, M. (2014) & 0 & 0 & 0 & 0 & 0 & 0 & 1 & 0 & 1 & 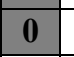 & 0 & 0 & 0 & 0 & 0 \\
\hline Chiva, O; Gil, J.; Hernando, C. (2014) & 0 & 0 & 0 & 0 & 0 & 0 & 0 & 0 & 1 & 0 & 0 & 0 & 0 & 0 & 0 \\
\hline Cruz, E. (2014) & 0 & 0 & 0 & 0 & 0 & 0 & 0 & 1 & 1 & 0 & 0 & 1 & 0 & 0 & 0 \\
\hline Davis, E (2006) & 0 & 1 & 0 & 0 & 0 & 0 & 0 & 0 & 1 & 1 & 0 & 0 & 0 & 0 & 0 \\
\hline Folco, M (2013) & 0 & 0 & 1 & 0 & 1 & 0 & 0 & 1 & 0 & 0 & 0 & 1 & 0 & 0 & 0 \\
\hline Forcadell, C. (2014) & 0 & 0 & 1 & 0 & 0 & 0 & 0 & 1 & 0 & 0 & 0 & 0 & 1 & 0 & 0 \\
\hline Fuguet, J (2014) & 0 & 0 & 1 & 0 & 0 & 0 & 1 & 0 & 0 & 0 & 0 & 0 & 1 & 0 & 0 \\
\hline García, T. (2011) & 0 & 0 & 1 & 0 & 0 & 0 & 0 & 1 & 0 & 0 & 0 & 0 & 1 & 0 & 0 \\
\hline Gaylord, N.; Chyka, D.; Lawley, G. (2012) & 0 & 0 & 0 & 0 & 0 & 1 & 1 & 0 & 0 & 0 & 0 & 0 & 1 & 0 & 0 \\
\hline Holgado, J. (2014) & 0 & 0 & 0 & 0 & 1 & 0 & 0 & 1 & 0 & 0 & 0 & 0 & 1 & 0 & 0 \\
\hline Iranzo, P. (2014) & 0 & 1 & 0 & 0 & 0 & 0 & 1 & 1 & 1 & 0 & 0 & 0 & 1 & 0 & 0 \\
\hline Knutson, K; Gonzalez, A. (2010) & 0 & 0 & 1 & 0 & 0 & 0 & 1 & 1 & 1 & 0 & 0 & 1 & 0 & 0 & 0 \\
\hline Konukman, F.; Schneider, R. (2011) & 0 & 0 & 0 & 0 & 0 & 0 & 0 & 0 & 0 & 0 & 0 & 0 & 0 & 0 & 1 \\
\hline Lake, V.; Jones, I. (2008) & 0 & 0 & 1 & 0 & 0 & 0 & 0 & 1 & 1 & 0 & 0 & 0 & 0 & 0 & 0 \\
\hline Lockhart, E. (2014) & 0 & 0 & 0 & 0 & 0 & 1 & 1 & 1 & 1 & 0 & 0 & 0 & 1 & 0 & 0 \\
\hline Maynes, N.; Hatt, B.; Wideman, R. (2013) & 0 & 0 & 1 & 0 & 0 & 0 & 0 & 1 & 0 & 0 & 0 & 1 & 0 & 0 & 0 \\
\hline Mc Connell, E.; Clasen, C.; Stolfi, A. (2010) & 0 & 0 & 0 & 0 & 1 & 1 & 1 & 1 & 0 & 0 & 0 & 1 & 0 & 0 & 0 \\
\hline Mirella, M. (2012) & 0 & 0 & 0 & 0 & 0 & 0 & 0 & 0 & 0 & 0 & 0 & 0 & 0 & 0 & 0 \\
\hline Páez, M.; Puig, J. (2013) & 0 & 0 & 1 & 0 & 0 & 0 & 0 & 1 & 0 & 1 & 0 & 0 & 0 & 0 & 0 \\
\hline Pakulski, L.A. (2011) & 0 & 0 & 0 & 0 & 0 & 1 & 1 & 0 & 1 & 0 & 1 & 0 & 0 & 0 & 0 \\
\hline Puig, J.; Gijón, M.; Martín, X. (2011) & 0 & 0 & 0 & 0 & 0 & 0 & 0 & 0 & 0 & 0 & 0 & 0 & 0 & 0 & 1 \\
\hline Roofe, N. L. (2011) & 0 & 0 & 0 & 0 & 0 & 1 & 1 & 0 & 0 & 0 & 1 & 0 & 0 & 0 & 0 \\
\hline $\begin{array}{l}\text { Sánchez, A.; Silió, J. A.; Sánchez-Raba, J. A. } \\
\text { (2012) }\end{array}$ & 0 & 0 & 0 & 0 & 0 & 1 & 1 & 0 & 1 & 0 & 0 & 0 & 1 & 0 & 0 \\
\hline Segarra, F. (2014) & 0 & 0 & 0 & 0 & 0 & 0 & 1 & 0 & 1 & 0 & 0 & 0 & 1 & 0 & 0 \\
\hline Téllez, A.; Eloy, J.; Sánchez, V. (2014) & 0 & 0 & 0 & 1 & 0 & 0 & 0 & 1 & 0 & 0 & 0 & 0 & 1 & 0 & 0 \\
\hline $\begin{array}{l}\text { Timpe, E. M.; Wuller, W. R.; Karpinski, J. P. } \\
\text { (2008) }\end{array}$ & 0 & 0 & 0 & 0 & 0 & 1 & 1 & 0 & 1 & 0 & 0 & 1 & 0 & 0 & 0 \\
\hline Vicens, P. (2014) & 0 & 0 & 1 & 0 & 0 & 0 & 0 & 1 & 1 & 0 & 0 & 0 & 1 & 0 & 0 \\
\hline $\begin{array}{l}\text { Winterbottom, Ch.; Vickie, E.; Lake, E. } \\
\text { (2013) }\end{array}$ & 0 & 0 & 1 & 0 & 0 & 0 & 0 & 1 & 0 & 0 & 0 & 1 & 0 & 0 & 0 \\
\hline
\end{tabular}


Lo anteriormente expuesto viene a justificar la presencia de este tipo de experiencias en contexto de vulnerabilidad social. Podemos ver en este sentido los trabajos de Folco (2013) y Holgado (2014) con niños o niñas que presentan algún tipo de discapacidad, o el trabajo de Chiva, Gil y Hernando (2014) focalizado en niños y niñas de 3-6 años desde la motricidad y el juego. En esta misma línea Téllez, Eloy y Sánchez (2014) se centran más en temas de género, otorgando otra perspectiva a la utilización de la metodología del A+SS.

En cuanto a experiencias en las que se muestre con claridad una intencionalidad de desarrollo de contenidos específicos con una metodología clara de investigación, las podemos encontrar vinculadas al ámbito de la salud, con un diseño experimental, cuasiexperimental o descriptivo y con un sesgo donde los objetivos de desarrollo personal y social quedan en un segundo plano (Gaylord, Chyka y Lawley, 2012;Mc Connell, Clasen y Stolfi, 2010; Pakulski, 2011; Roofe, 2011; Timpe, Wuller y Karpinski, 2008). Es evidente que la tradición positivista de las ciencias de la salud impone un diseño de investigación en el que podemos o no estar de acuerdo, pero sí debemos reconocer como posibilidad de avance en la implementación y evaluación de experiencias de A+SS.

En la misma línea anterior, pero sin entrar en el espacio de la formalidad de la investigación, también encontramos trabajos que abordan la metodología con una clara intencionalidad de mejora de competencias disciplinares en el estudiante universitario. Tenemos en esta línea los trabajos de Sánchez, Silió y Sánchez-Raba (2012) centrado en la incorporación de las Tecnologías de Información y la Comunicación al aula de Educación Infantil, los trabajos de Forcadell (2014) en el ámbito de la educación emocional y de Lockhart, E. (2014), Iranzo, P. (2014a y b) y Fuguet, (2014), en comunicación lingüística y enseñanza de la lecto-escritura.

En cuanto el abordaje de este tipo de proyectos desde una metodología cualitativa, tenemos varios autores que lo declaran, aunque de la declaración a la realidad puede haber ciertas diferencias. Falta una clara definición de este tipo de metodología en los trabajos y prácticamente se limitan a identificar método y definir instrumentos, sin encarar lo importante, el proceso de producción y análisis de la información. A pesar de lo anterior 
podemos citar los trabajos para su revisión de Bartolomé (2013), Capella, Gil y Martí (2014) y Capella, Gil y Martí (2015), destacando en esta línea, por ser el que mejor muestra la metodología desarrollada, el de Davis (2006).

De cara a fundamentar y profundizar en el A+SS, es recomendable revisar los trabajos de Aramburuzabala y García (2012), que realizan una retrospectiva muy interesante sobre el tema, aunque desde una perspectiva global y no centrada exclusivamente en la formación de docentes de Educación Infantil. En la misma línea pero con una mirada más crítica e interdisciplinar de lo que debe suponer la puesta en marcha de esta metodología, recomendamos la revisión del trabajo de Amat y Moliner (2010). Por último, la revisión del trabajo de Puig, Gijón y Martín (2011) nos adentrará en un análisis del $\mathrm{A}+\mathrm{SS}$ desde la óptica que podríamos entender como básica en este tipo de intervenciones con el alumnado universitario: la educación para la ciudadanía, que encerraría uno de los grandes sentidos de llevar esta metodología al aula.

\section{Conclusiones}

Podemos incidir en que la rotura del modelo vertical de hacer ciencia y la incorporación de metodologías más participativas, hace que el A+SS encuentre un sustento importante para comenzar a definir otra forma de generar conocimiento, en el que las problemáticas de la Educación Infantil busquen soluciones, si no generalizables, al menos transferibles a otros contextos de similares características. Construir para y con la comunidad es la clave, desde sus intereses y su lenguaje.

Queda claro que hay una barrera significativa entre hacer voluntariado frente a construir un proceso de aprendizaje guiado desde el Aprendizaje y Servicio Solidario. Estamos hablando de pasar del ejercicio de la responsabilidad social a la puesta en marcha en el aula universitaria de una metodología que, además de incorporar al estudiante al contexto, pretende provocar aprendizajes específicos, sistémicos y prosociales de la materia, desde la disonancia cognitiva que estimula el trabajo desde la realidad.

En cuanto al estado de cuestión que hemos pretendido desarrollar en el artículo, decir en primer lugar que podemos hablar de un buen nivel de experiencias que pueden ser 
orientativas para el lector, pero escasa investigación que nutra, tamice y enriquezca las futuras propuestas de A+SS en el ámbito de la formación del docente de Educación Infantil. Se adolece de publicaciones estables sobre la temática, de autores de referencia en investigación, que no en cuanto a la realización de experiencias, de escaso bagaje de trabajos y especialmente de pautas que ayuden a guiar futuras investigaciones.

Aunque se vislumbran objetivos claros, orientados fundamentalmente al desarrollo de competencias inter e intrapersonales, y en menor medida las de carácter específico y sistémico, sí se produce un cierto acuerdo en dar importancia a los beneficios y mejoras de la comunidad. Evidentemente queda mucho camino por recorrer, especialmente en cuanto a consolidar los diseños de investigación y las técnicas a utilizar para producir la información.

Aún es pronto para ver el alcance e impacto del A+SS como metodología en la formación de docentes, prácticamente las fuentes de información pertenecen a estos últimos cinco años, pero sí estamos en disposición de decir que se presenta un futuro prometedor, especialmente ante las demandas de los estudiantes que buscan un aprendizaje desde el 'hacer', más autónomo y donde el docente comience a aceptar un papel más de mediación que de referente del conocimiento.

\section{Referencias bibliográficas}

Amat, A. F. y Moliner, L. (2010). El Aprendizaje Servicio en la Universidad: una estrategia en la formación de ciudadanía crítica, REIFOP, 13, 4.

Aramburuzabala, P. y García, P. (2012). El aprendizaje-servicio en la formación de maestros, VII Congreso de la CIDUI. Tarragona.

Bartolomé, S. J. (2013). Growing Through Service: Exploring the Impact of a ServiceLearning Experience on Preservice Educators, Journal of Music Teacher Education, 23, 1, 79-91.

Campo, L. (2014). Aprendizaje servicio y educación superior. Una rúbrica para evaluar la calidad de proyectos. Tesis doctoral. Universitat de Barcelona. Consultada el 15 de abril de 2015, disponible en: 
http://www.tesisenred.net/bitstream/handle/10803/277560/01.LCC_TESIS.pdf?seq uence $=1$

Capella, C., Gil, J. y Martí, M. (2014). La metodología del aprendizaje-servicio en la educación física, Apunts, 116, 33-43.

Capella, C., Gil, J. y Martí, M. (2015). Estudio de caso múltiple con historias de vida en el grado de educación infantil: aprendizaje-servicio en la didáctica de la educación física. Profesorado: Revista de curriculum y formación del profesorado, 19, 1, 334348.

Carr, W. y S. Kemmis (1988). Teoría crítica de la enseñanza. La investigación-acción en la formación del profesorado. Barcelona: Martínez Roca.

Cruz, E. (2014). Trabajo Fin de Grado Cuentos, Universitat Rovira i Virgili, http://www.urv.cat/aprenentatgeservei/es_infanil_resum.html\#societat

Chiva, O, Gil, J. y Hernando, C. (2014). Innovación metodológica en la universidad. Aprendizaje servicio en la didáctica de la expresión corporal y los juegos motrices. Tándem, Didáctica de la Educación Física, 44, 41-48.

Davis, E (2006). Characterizing productive reflection among preservice elementary teachers: Seeing what matters. Teaching and Teacher Education, 22, 281-301.

Ebbutt, D. y J. Elliott (1990). ¿Por qué deben investigar los profesores? En J. Elliott, La investigación-acción en educación (pp. 176-190). Madrid: Morata.

Folco, M. (2013). Estrategias docentes en la inclusión de niños y niñas en situación de vulnerabilidad. Revista internacional de educación para la justicia social, 2, 255270.

Forcadell, C. (2014). Intervención psicoeducativa en las dificultades emocionales y de relación, Universitat Rovira i Virgili, http://www.urv.cat/aprenentatgeservei/es_infanil_resum.html\#societat

Freire, P. (1990). La naturaleza política de la educación: cultura, poder y liberación. Barcelona: Paidós. 
Fuguet, J. (2014). La escuela de educación infantil LECIT, Universitat Rovira i Virgili, http://www.urv.cat/aprenentatgeservei/es_infanil_resum.html\#societat

García, T. (2011). Aportaciones ciudadanas desde el aprendizaje servicio: universidad, escuela y comunidad conectadas. Revista Interuniversitaria de Formación del Profesorado, 71, 125-141.

Gaylord, N., Chyka, D. y Lawley, G. (2012). Developmental Evaluation of Preschool Children: A Service-Learning Experience for Nursing, Students, Journal of Nursing Education, 51, 12, 710-713.

Gibbons, M., Limoges, C., Nowotny, H., Schwartzman, S., Scott, P. y Trow, M. (1994). The new production of knowledge. The dinamics of science and research in contemporary societies. London: SAGE Pub.

Gollete, G. y Lessard-Hébert, M. (1988). La investigación-acción. Sus funciones, su fundamento y su instrumentalización. Barcelona: Laertes.

Herrero, A. (2010). Una nueva forma de producción de conocimientos: el aprendizajeservicio en educación superior. TZHOECOEN. Revista Científica. 3, 5, 63-80.

Holgado, J. (2014). Sociedad, familia y educación, Universitat Rovira i Virgili, http://www.urv.cat/aprenentatgeservei/es_infanil_resum.html\#societat

Iranzo, P. (2014 a). Enseñanza y aprendizaje de lenguas y lectoescritura I, Universitat Rovira i Virgili, http://www.urv.cat/aprenentatgeservei/es_infanil_resum.html\#societat

Iranzo, P. (2014 b). Enseñanza y aprendizaje de lenguas y lectoescritura II, Universitat Rovira i Virgili, http://www.urv.cat/aprenentatgeservei/es_infanil_resum.html\#societat

Israel, B., Schultz, A., Parker, E. y Becker, A. (1998). Review of community-based research: Assesing partnership Approaches to improve Public Health. Annual Rev. Public Health, 19, 173-202. 
Jacoby, B. y Associates (1996). Service-learning in Higher Education. Concepts and practices. San Francisco: Jossey Bass.

Kemmis, S. y McTaggart, R. (1988). Cómo planificar la investigación acción. Barcelona: Alertes.

Kendall, J. (coord). (1990). Combining service and learning. The Journal of General Education, Vol. 40 (1991), pp. 292-294 Knutson, K y Gonzalez, A. (2010). Domestic and international service learning experiences: A comparative study of pre-service teacher outcomes, Issues in Educational Research, 20, 1.

Lake, V. y Jones, I. (2008). Service-learning in early childhood teacher education: Using service to put meaning back into learning. Teaching and Teacher Education, 24, 2146-2156

Lewin, K. (1946). Action Research and Minority problems. Journal of Social Issues, 2, 4, 34-46.

Lockhart, E. (2014). Competencia comunicativa oral y cultura popular, Universitat Rovira i Virgili, http://www.urv.cat/aprenentatgeservei/es_infanil_resum.html\#societat

Maynes, N., Hatt, B. y Wideman, R. (2013). Service Learning as a Practicum Ex, Canadian Journal of Higher Education, 43, 1, 80-99.

Mc Connell, E., Clasen, C. y Stolfi, A. (2010). Community service and the pediatric exam: An introduction to clinical medicine via a partnership between first year medical students and a community elementary school. Teaching and Learning in Medicine, $22,3,187-190$.

Páez, M. y Puig, J. (2013). La reflexión en el aprendizaje-servicio. Revista Internacional de Educación para la Justicia Social (RIEJS), 2, 2, 13-32.

Pakulski, L. A. (2011). Addressing qualified personnel shortages for children who are deaf or hard of hearing with an interdisciplinary service learning program. American Journal of Audiology, 20, 2, 203-219. 
Puig, J. M. (coord.) (2009). Aprendizaje servicio (ApS). Educación y compromiso cívico. Barcelona: Graó.

Puig, J., Gijón, M. y Martín, X. (2011). Aprendizaje-servicio y Educación para la Ciudadanía. Revista de Educación, extr., 45-67.

Puig, J. M. y Palos, J. (2006). Rasgos pedagógicos del aprendizaje servicio. Cuadernos de pedagogía, 357.

Roofe, N. L. (2011). Improving families' nutrition knowledge through service learning. Allied Health, 40, 4, 194-198.

Sánchez, A., Silió, J. A. y Sánchez-Raba, J. A. (2012). Maestros y estudiantes de maestro, juntos para una escuela más digital: una experiencia de aprendizaje servicio. Aula de innovación educativa, 217, 38-42.

Service learning 2000 center (1996). Service Learning Quadrants. Palo Alto, California.

Stanton, T. (1990). Service Learning: Groping Toward A Definition, in J. Kendall (coord) Combining service and learning. Raleigh, National Society for Internships and Experiential Education.

Tapias, M. N. (2010). La propuesta pedagógica del "aprendizaje-servicio": una perspectiva latinoamericana. TZHOECOEN. Revista Científica, 3, 5, 23-43.

Téllez, A., Eloy, J. y Sánchez, V. (2014). Género y diversidad en la educación. La interacción de mujeres con capacidades diferenciadas (mujeres no estándar) en un Centro Público de Educación Infantil y Primaria. Cuestiones de género: de la igualdad y la diferencia, 9, 63-88.

Timpe, E. M., Wuller, W. R. y Karpinski, J. P. (2008). A regional poison prevention education service-learning project. American Journal of Pharmaceutical Education, 72, 4-87.

Vicens, P. (2014). Trabajo Fin de Grado - Practicum Cooperación,Universitat Rovira i Virgili, http://www.urv.cat/aprenentatgeservei/es_infanil_resum.html\#societat 
Winterbottom, Ch., Vickie, E. y Lake, E. (2013). Fostering Social Justice through ServiceLearning in Early Childhood Teacher Education. Revista Internacional de Educación para la Justicia Social (RIEJS), 2, 2, 33-53. 